\title{
VALORES DE NOMÁS EN EL ESPAÑOL ANDINO
}

\author{
JOANNA WILK-RACIĘSKA \\ Universidad de Silesia
}

Resumen

El objetivo del artículo es presentar un análisis de los valores del vocablo nomás en el español andino realizado desde la perspectiva de la lingüística cultural (Anusiewicz, 1994; Palmer, 2000; Wilk-Racięska 2012 y 2018), una metodología multidimensional que, utilizando las herramientas de la semántica, de la pragmática y del cognitivismo, permite estudiar las relaciones entre el español de América con las lenguas sustráticas desde el punto de vista de las visiones del mundo enraizadas en las estructuras lingüísticas. La autora esboza la metodología, define las nociones y herramientas básicas y, por último, ilustra la presentación con un breve análisis de las motivaciones y evolución semántico-pragmático-cognoscitivas del adverbio nomás en el español andino.

Palabras clave: español andino, quechua, sustrato, análisis semántico-pragmático, motivaciones cognitivas, adverbio.

\section{VALUES OF ANDEAN SPANISH NOMÁS}

\section{Abstract}

The aim of this article is to present an analysis of different values of Andean Spanish adverb nomás, conducted by means of methodology referred to as cultural linguistics (Anusiewicz, 1994; Palmer, 2000; Wilk-Racięska, 2012 and 2018), understood as a multidimensional research system which uses various tools, depending on the phenomenon under the investigation. Hence, the research apparatus of cultural linguistics covers semantic, cognitive and pragmatic tools. This methodology allows for the thorough examination of the relations between Andean Spanish and its substrative languages. The autor introduces key notions, defines methodology and presents a practical ilustration of the hypothesis put forward in the form of a short analysis of some values of the Spanish Andean adverb nomás.

Keywords: Andean Spanish, Quechua, substrative languages, semántico- pragmatic analysis, cognitive motivations, adverb. 


\section{INTRODUCCIÓN}

Actualmente tres son los aspectos básicos del estudio de la(s) variante(s) andina(s) del español: el aspecto sociolingüístico, el didáctico y el lingüístico. Dentro de esta última índole las investigaciones giran, ante todo, en torno a los cambios morfosintácticos y lexicales respecto al castellano peninsular, considerado estándar. Los estudiosos suelen adscribir los cambios a los calcos y/o las influencias de las gramáticas de las lenguas sustrato, o bien ver en ellos la supervivencia de las formas y/o usos del castellano de los tiempos de la conquista y, por último, hay muchos que optan por la convergencia. Es natural, que los hablantes andinos hayan acomodado algunas de sus prácticas idiomáticas a las condiciones de la lengua adoptada. Tampoco puede descartarse la importancia de la convergencia. Todos estos aspectos del español del área andina se encuentran investigados y documentados en los trabajos de ilustres estudiosos del tema, como Adelaar (1977), de Granda (1999 y 2001), Cerrón Palomino (2004) o Calvo Pérez (2000), por mencionar los más conocidos.

\section{OBJETIVO DEL ESTUDIO}

En el estudio presente nos interesan ante todo los motivos y efectos de la transferencia. Apoyándonos en las evidencias ya existentes nos dedicamos, por un lado, a buscar y comentar las motivaciones conceptuales que propiciaron algunos cambios lingüísticos y pragmáticos de las variedades del español estudiadas y, por el otro, a analizar las razones semánticas que han permitido estos cambios en el castellano. En primer lugar, hemos supuesto que una buena parte de los cambios en cuestión se debe a la necesidad de expresar en la lengua adoptada algunos elementos de la visión lingüística del mundo indígena (ver infra) propios de la cultura y mentalidad amerindia. Por otra parte, hemos supuesto también que la adaptación de las construcciones lingüísticas castellanas a los objetivos de la visión lingüística amerindia se ha fundamentado en la adecuación de los conceptos primarios encerrados en su estructura semántica (Wilk-Racięska, 2020a). Nuestra hipótesis se sustenta, ante todo, en la enorme diferencia que presentan las visiones lingüísticas del mundo amerindias y la occidental, y en la convicción de 
que tales distinciones deberían haberse reflejado en el español de la zona que se había ido configurando a través de los siglos partiendo de esta contrariedad. Al mismo tiempo, creemos que por más distintas que sean dos visiones lingüísticas del mundo, las lenguas naturales se fundamentan en un nú-mero de conceptos semánticos primarios universales íntimamente ligados a la naturaleza humana que permiten descifrar tanto las nociones que las lenguas sustrato han introducido en el español, como los elementos semánticos propios de algunas estructuras castellanas que han permitido adoptarlas para expresar en la lengua adoptada la visión del mundo indígena. Por estos motivos, para los fines del estudio presente adoptamos las herramientas de la lingüística cultural (que ya definimos en Wilk-Racięska, 2007, 2009, 2012 y 2016).

\section{METOdOLOGÍA Y DEFINICIONES}

Es bien sabido que un análisis unidimensional (funcional, semántico, pragmático, etc.) aplicado a las investigaciones relacionadas con el cambio lingüístico en situaciones de contacto no está en condiciones de agotar todas las cuestiones relativas al tema. Por este motivo, en este estudio nos serviremos de la metodología de la lingüística cultural (Wilk-Racięska, 2016), definida como una disciplina lingüística multidimensional, la cual permite analizar las estructuras lingüísticas de las lenguas naturales desde tres fundamentales puntos de vista: el conceptual (semántico), el pragmático y el cognitivo, ofreciendo de este modo una descripción más completa de las construcciones lingüísticas en el uso. Los métodos de la lingüística cultural se originan en distintas ramas científicas. No obstante, la variante que proponemos enfoca tres perspectivas lingüísticas principales: la semántica, que descifra la estructura conceptual de las expresiones lingüísticas y constituye el andamiaje mental de cada lengua natural, la pragmática, que propicia el análisis de las funciones discursivas de estas expresiones; y la cognitiva, que facilita el entendimiento de las motivaciones cognitivas de su uso y evolución ${ }^{1}$. El empleo de las herramientas pertenecientes a

${ }^{1}$ La presentación exhaustiva de la metodología propuesta y de todas sus herramientas se encuentra en Wilk-Racięska (2016). En este artículo utilizaremos tan solo tres herra- 
cada uno de estos enfoques es posible gracias a la interdependencia entre las funciones discursivas, la motivación cognitiva y la estructura semántica, la cual, por su parte, está íntimamente vinculada con la visión del mundo ${ }^{2}$ que cada lengua natural refleja (Wilk-Racięska, 2012 y 2016).

En el aparato de la lingüística cultural, la herramienta fundamental empleada en la primera etapa de la investigación es la descomposición conceptual en conceptos simples. Entendemos por conceptos simples (términos primarios o indefinibilia) los conceptos que se definen intuitivamente (Bogacki y Karolak, 1991; Karolak, 1994) ${ }^{3}$. La complejidad del contenido semántico de un lexema raras veces se manifiesta en su forma superficial. Las estructuras conceptuales pueden encerrar otras estructuras conceptuales más o menos complejas, pero todas ellas se fundamentan en conceptos simples. Esto significa que todas las estructuras conceptuales pueden descomponerse en conceptos cada vez más simples hasta llegar a los conceptos primarios.

\subsection{Aspectualidad}

Al hablar de las lenguas sustráticas del español andino no se puede olvidar que las lenguas amerindias no son lenguas temporales (como por ejemplo, el español) sino aspectuales. Ello significa que la disposición de los eventos en el tiempo como continuos o limitados de algún modo es para estas lenguas un factor prioritario, mientras que las len-

mientas: la descomposición semántica (nivel conceptual), la inferencia pragmática y el principio de iconicidad (nivel cognitivo), cuyas definiciones se explicarán a lo largo del estudio.

${ }^{2}$ Por la visión del mundo entendemos un sistema de conocimientos socio-filosóficos, creencias, ritos etc., mientras que la visión lingüística del mundo es una estructura conceptual grabada en las propiedades gramaticales y léxicas de una lengua dada, que se realiza en la enunciación (Grzegorczykowa, 1999). La visión lingüística del mundo es, junto al arte, la música, etc., una de las formas de expresar la visión del mundo general representada por una comunidad sociolingüística dada. Dicho en otras palabras, cada lengua natural sirve para expresar la visión del mundo propia de la comunidad sociolingüística que la creó.

${ }^{3}$ La definición que aquí empleamos, siguiendo a Bogacki y Karolak (1991), está enraizada en la misma idea leibniziana que la metalengua de Wierzbicka (1988 y 1991), pero en el modelo de Wierzbicka las paráfrasis se realizan en la primera persona del singular, hecho que no siempre resulta apropiado para el análisis de las visiones del mundo no orientadas al egocentrismo (véase también Palmer, 2000). 
guas orientadas al tiempo la consideran como secundario. Desde un punto de vista gramatical, ello significa que las lenguas orientadas al tiempo han desarrollado el sistema de los tiempos gramaticales asociados al verbo, mientras que muchas lenguas orientadas al aspecto (muchas lenguas eslavas, amerindias o semíticas, etc.) lo han limitado. Además, muchas lenguas amerindias son aglutinantes y hasta polisintéticas $^{4}$, con un orden de encadenación de los morfemas entre sí bastante libre y el concepto de aspecto no necesariamente ligado a los lexemas verbales (Liuzzi y Kirtchuk, 1989). En consecuencia, las estructuras conceptuales representadas por los morfemas amerindios son complejas y su manejo formal y pragmático resulta, en muchos casos, bastante peculiar desde el punto de vista de las gramáticas occidentales. Las estructuras lingüísticas de las lenguas indígenas lexicalizan muchos parámetros distintos de los que las visiones lingüísticas del mundo occidental consideran importantes. Por estos motivos, a lo largo de nuestras investigaciones, entendemos la aspectualidad como una dimensión semántico-discursiva fundamentada en dos conceptos simples: el de discontinuidad (puntualidad) y el de continuidad (duratividad) ${ }^{5}$. Estos conceptos pueden funcionar como aspectuales sirviendo, en tal caso, para enfocar la manera en que el hablante percibe el evento relacionado: como concluido o no concluido, respectivamente (Wilk-Racięska, 2020b). Dentro del marco de su función aspectual ambos conceptos pueden configurarse. Ello significa que a fin de presentar el evento como, por ejemplo, incoativo, es decir, enfocar su comienzo, podemos combinar dos expresiones con uno de los conceptos opuestos encerrado en cada una de ellas, de tal modo que el lexema con el concepto discontinuo domine el que expresa el concepto continuo limitándolo de la izquierda, es decir, enfocando el principio del evento denotado. En español son las perífrasis denominadas aspectuales (ponerse a + infinitivo, por ejemplo) las que sirven para expresar estos valores combinados,

${ }^{4}$ Ya Tovar (1964) observa que por ejemplo en el estudio del quechua hay que subrayar la subordinación de los sufijos acumulados; mientras que, en el guaraní, el mataco, y en mayor medida en el cuna, destacan los rasgos incorporantes. También es importante la escasez o falta de morfemas para indicar el tiempo verbal.

${ }^{5}$ Nuestro planteamiento se ha inspirado fuertemente en la teoría del aspecto formulada en el marco de la sintaxis semántica de Karolak (1994) y ésta en la hipótesis de Guillaume (1970). 
mientras que las lenguas orientadas al aspecto se valen ante todo de los afijos o morfemas especiales. Sin embargo, también hay casos en los cuales el rol de los conceptos en cuestión resulta más discursivo que semántico, aspectual. En tales situaciones la puntualidad o la continuidad promueven otros valores. Visto esto, en nuestro estudio no tomamos en consideración la división formal entre aspecto y modo de acción, puesto que conforme al planteamiento adoptado ambos tipos se fundamentan en los conceptos semánticos. Todo ello no significa que descartemos la necesidad de formular y operar con los términos aspecto gramatical, flexivo, sintagmático para los fines formales, es decir, en cuanto al modo de expresar los valores aspectuales mediante las formas gramaticalizadas, sino que el término aspecto verbal y lo que sigue, aspecto léxico relacionado con los verbos como portadores exclusivos del valor en cuestión, ya no se adecua a todos los sistemas amerindios. Recordemos que muchas lenguas andinas revuelven el «buen orden y disposición" del lugar de continuidad y discontinuidad asentado en nuestro sistema conceptual -véase, por ejemplo, Adelaar (1977) y Calvo Pérez (2000)-.

\subsection{Una aproximación a los morfemas amerindios llamados aspec- tuales}

La morfología de las lenguas amerindias cuenta con numerosos afijos que, en muchos casos, representan conceptos no lexicalizados o lexicalizados en un grado mucho menor en la lengua española. Aunque el aspecto es obviamente uno de estos conceptos lexicalizados en los morfemas del quechua y otras lenguas amerindias, hay otros valores también muy importantes cuya investigación puede contribuir a nuestro conocimiento de la visión del mundo amerindio. Recordemos las palabras de los autores de la página web Quechua en Cochabamba ${ }^{6}$, quienes empiezan la presentación de los sufijos con las palabras siguientes:

Las palabras castellanas escritas inmediatamente debajo de los sufijos quechuas, en ciertos artículos de esta lista, de ninguna manera pueden ser consideradas como traducciones. Se trata solo de

\footnotetext{
${ }^{6}$ Última modificación: 30 de agosto de 1996. Disponible en: $<$ http://jlancey.tripod.com/Peda/Suffixes.htm> [consulta: 17 de febrero de 2019]).
} 
equivalentes, que hemos indicado para hacer más fácil su consulta. Se debe enfatizar que ningún sufijo quechua puede ser traducido de un modo correcto sin considerar cuidadosamente el contexto en que aparece.

Es de subrayar, que la dominación en los estudios aspectológicos de las discusiones sobre el estatus gramatical del aspecto en los morfemas en cuestión (lo que, dicho sea de paso, no se puede negar desde el punto de vista morfológico) ha apartado a un segundo plano la cuestión de la complejidad de las estructuras conceptuales de aquellos morfemas y las posibilidades de poner de relieve uno u otro de los componentes de su contenido. Ello provocó una extensión del término aspectual a cada morfema que en su estructura conceptual encierre también uno de los dos conceptos, el continuo o el discontinuo, sea su función en una situación contextual dada realmente aspectual o no. Este es el caso de los morfemas de las lenguas andinas, en cuyas estructuras los conceptos tanto aspectuales como los de otra índole se solapan y configuran de maneras diferentes. Subrayemos, sin embargo, que todos estos usos están reunidos bajo la etiqueta de aspecto. Ya Adelaar (1977) observa que los sufijos aspectuales se emplean para marcar características muy variadas. No obstante, las evidencias facilitadas tanto por los investigadores como por las gramáticas de quechua testimonian a favor de una cierta complejidad de las estructuras de los morfemas en cuestión, la cual permite diferentes y focalizaciones contextuales.

\section{UN EJEMPLO DEL ANÁLISIS: NOMÁS}

Veamos ahora un ejemplo del análisis de la evolución pragmáticogramatical en el español andino que consideramos interesante para los objetivos de este artículo: el caso de nomás. Siguiendo la metodología y tomando en cuenta todas las definiciones esbozadas supra analicemos la estructura conceptual reflejada por nomás y presentaremos un esbozo de la evolución semántico-pragmática del adverbio y sus supuestas motivaciones. Este caso nos parece especialmente interesante porque el adverbio nomás es muy frecuente en casi toda el área de América Latina, aunque no en el español peninsular. Algunos investigadores (Calvo Pérez, 2000; Gutiérrez González, 2011) lo consideran proveniente de la 
contracción del negativo no y el aditivo más con el significado original de nada más. También Kanny (1976: 367) advierte que "[e]n Hispanoamérica se ha conservado el uso arcaico de no más y extendido su valor a acepciones desconocidas en España. En España se prefiere nada más». En el uso americano, sin embargo, el adverbio presenta valores distintos de su «original» español.

\subsection{Estructura conceptual de nomás}

Empecemos por analizar la estructura nocional del adverbio en búsqueda de conceptos primitivos (el nivel básico del análisis multidimensional). Si originalmente la construcción representa negación + adición, podemos arriesgar una suposición de que en esta fase primaria se trate de una estructura conceptual en la cual estamos ante la operación de agregar (= adición) puesta en el alcance de la negación. El concepto metalingüístico de negación pertenece a los conceptos simples pero la operación de agregar (= adición) presenta ya un grado más alto de la complejidad, es decir, forma un esquema experiencial. Como todo esquema, la operación de agregar (= adición) presenta un proceso (en el sentido langackeriano) no anclado ni en el tiempo ni en el espacio con posiciones de argumentos abiertas. El sentido básico, experiencial, de adición es agregar una cosa a otra. La acción de agregar, sin embargo, ya es un evento que podemos considerar perfectivo si lo conceptualizamos como un acto, una acotación (boundedness) seleccionada de adición. También podemos conceptualizarlo como iterativo, si tenemos en cuenta los actos de adición repetidos de modo continuo, sin que los momentos principal y terminativo pertenezcan a su perfil ${ }^{7}$, es decir, cuando la continuidad del evento es conceptualizada como no limitada por ningún lado. Vemos entonces que, cualquiera que sea su interpretación aspectual final, el esquema de adición como parte integral de la estructura de no / nada más en su valor primario contiene de manera

\footnotetext{
${ }^{7}$ Recordemos brevemente que «[s]egún Langacker [...], una expresión, cualquiera que sea su complejidad, adquiere su significado imponiendo un perfil a una base. La base se puede definir como la matriz subyacente de dominios cognitivos relevantes que se requiere o se evoca para comprender una expresión determinada. El perfil, por su lado, es la subestructura destacada sobre la base que la expresión en cuestión designa conceptualmente. Esta asimetría es análoga a la noción de figura y fondo de la psicología de la forma, puesto que la base actúa como trasfondo para el perfil» (Cuenca y Hilferty, 1999: 76).
} 
necesaria el concepto de discontinuidad que permite acotar el (los) acto(s) de adición. Observemos también, que siguiendo la teoría experiencial podemos decir que la vinculación entre el concepto de discontinuidad y el de negación sirve aquí para limitar la adición de objetos, relaciones, etc.

Pasemos ahora al segundo nivel del análisis multidimensional, el cual nos permitirá descubrir los valores pragmáticos de la expresión castellana. Analizando los valores del vocablo desde el punto de vista pragmático es posible presentarlo, en algunos contextos, en categorías de presuposición/inferencia: no quiero nada más; no hagas nada más; no digas nada más. Lo que se presupone en estos enunciados es la existencia de una cantidad de objetos reunidos/eventos efectuados, etc., mientras que lo que se explicita y enfoca es la limitación desde el polo derecho (es decir, la determinación del punto final más allá del cual ya no pueden seguir avanzando) que, gracias a la negación y a la función aspectual terminativa de concepto de discontinuidad, entra en el perfil del evento denotado por el enunciado. En suma, ambas interpretaciones se fundamentan en el contenido conceptual del vocablo.

\subsection{Evolución semántico-pragmática de nomás en el español andino}

La estructura nocional de la construcción analizada que presenta una configuración de conceptos bastante compleja es, pues, el punto de partida para una evolución semántico-pragmática del vocablo. También es importante tener en cuenta que durante este proceso de evolución el adverbio ha sufrido, además de las modificaciones del valor y las ortográficas, un desplazamiento dentro del enunciado a la posición posverbal.

Según la Real Academia Española, el adverbio nomás en América Latina tiene tres acepciones, que podemos considerar extensiones de su significado en el uso motivadas cognitivamente:

1.adv. m. Am. Mer., C. Rica, Hond., Méx. y Nic. no más (solamente); 2. adv. m. Am. Mer., El Salv., Hond. y Méx. U. en oraciones exhortativas, generalmente pospuesto, para añadir énfasis a la expresión. Pase nomás. Atrévase nomás; 3. adv. m. Arg., Bol., Col., El Salv., Nic. y Ur. Apenas, precisamente (RAE, 2019). 
Los usuarios de distintas variantes del español de América registran también: 4. el valor irónico: [...] Seguí nomás dejando todo tirado, total acá está tu sirvienta para ordenar (Alepsi-Argentina) /.../ Sigue así nomás sin arreglar el problema, total para eso estamos el resto (BetildusChile) y 5. el valor de amenaza: ¡Sigue bebiendo nomás y ya verás como terminas botado en la calle (Betildus-Chile) ${ }^{8}$. Mientras tanto, en la opinión de Calvo, nomás en su forma y uso actual pertenece (con pero y pues) a la clase de partículas restrictivas (Calvo Pérez, 2000: 95-102).

Muchos lingüistas (Cusihuamán, 1976; Calvo Pérez, 2000; entre otros) proponen considerar los valores de nomás en el área del alcance del quechua y del aymara como homólogos a algunos valores de las partículas /$\mathrm{lla} /$ en quechua y $/-\mathrm{k}(\mathrm{y}) /{ }^{9}$ en aymara. Calvo Pérez (2000: 101) advierte que la función más destacable de las partículas indígenas en cuestión parece ser la del marcador de limitación exclusiva. Vamos, pues, a esbozar los valores de estas dos partículas. A nuestro parecer, de los ejemplos de Cusihuamán (1976) y Calvo Pérez (2000) -resulta, en primer lugar, que las partículas /-lla/ y /-k(y)/ no contienen el valor presuposicional presente en la construcción española no/nada más-. Las partículas indígenas parecen funcionar como focos reales que sacan a la luz un solo comunicado dejando el resto de los significados/informaciones eventuales en la oscuridad. Calvo cita el ejemplo de machullaña explicando que este vocablo «significa literalmente el que ya es viejo no más, como si el resto de las cosas que puedan afectar a la persona en concreto no importaran» (Calvo Pérez, 2000: 85). Vemos, pues, que los elementos acotados por el alcance de /-lla/ no contrastan con ningún otro elemento del discurso que pudiera entrar en juego; otros elementos -por si existen- no se toman en consideración. Otro valor de las partículas analizadas distinguido por este estudioso, es el de la restricción de referencia: nayakiwa = yo no más/sólo yo. En nuestra opinión, en estos dos casos no parece tratarse de dos valores diferentes, sino de la diferencia del estatus del elemento enfocado ya que

8 Ejemplo tomado del foro «Entren nomás -encuesta para españoles» (en línea: $<$ http://forum.wordreference.com/showthread.php?t=572796> [consulta: 14 de enero de 2019]); en adelante, «foro».

${ }^{9}$ Para no exceder los límites del estudio y teniendo en cuenta que el quechua es mucho más extendido que el aimara, en este artículo nos interesa solamente la partícula quechua /-lla/ 
en ambos casos citados estamos ante el valor limitativo: en el primer caso, se enfoca un objeto nocional y, en el otro, uno real.

Sin embargo, lo que más importa es la cualidad de exclusión que observan tanto Calvo Pérez (2000:101) como Cusihuamán (1976). Para Cusihuamán, /-illa/ con el valor de la limitación exclusiva puede ser traducido como no más, sólo, únicamente: Paqari-lla-ntawanñan kaypi kasaq = estaré aquí sólo ya el día el de mañana. La traducción con sólo es una opción natural puesto que solo es un exponente del concepto de unicidad, así que el uso de la extensión de su significado (sólo) tiene por objetivo marcar una acotación exacta del perfil del elemento denotado. No obstante, como señalan los autores citados, las traducciones con nomás ${ }^{10}$ también aparecen sin que se pierda el valor de la limitación exclusiva de /-illa/.

Como es bien sabido nomás, en algunos de sus usos en el español americano, también sirve para acotar de manera exclusiva el elemento denotado:

(1) Nomás así entiende la gente (Gutiérrez-González, 2010: 42, español rioplatense)

(2) ¡Nomás queda una cerveza en el refri! («Foro»; ArgótideMéxico)

donde (1) significa Sólo así entiende la gente/no hay otra manera de entenderlo y (2) Queda una sola cerveza/no hay más cervezas.

La más interesante de las descripciones de este valor de nomás aparece, a nuestro parecer, en los trabajos de Gutiérrez-González (2011), quien estudia el español rioplatense. La autora considera nomás el operador focal sintético y, más precisamente, el foco contrastivo exhaustivo que, «además de mostrar un contraste, establece una lectura de unicidad, es decir, niega todas las alternativas posibles en el universo del discurso excepto una» (Gutiérrez-González, 2011: 626). Coincidimos

${ }^{10}$ Gutiérrez-González (2011: 42) facilita unos ejemplos que comparan el uso de nomás con el del sólo peninsular, por ejemplo: Me regaló (nomás/sólo) la toalla (nomás/`sólo), ninguna cosa más. 
con esta autora en que en los ejemplos citados el adverbio establece una lectura exhaustiva del elemento enfocado. Observemos que este valor de acotador exclusivo se perfila tan sólo cuando el adverbio se antepone al elemento focalizado y tales usos no son muy típicos, ya que se observa una tendencia a posponer este adverbio a la parte constituyente del enunciado. Comparemos: (1) Nomás así entiende la gente y (3) El supermercado está acá nomás El adverbio nomás no representa el concepto de unicidad, el cual sí es propio de sólo, pero su estructura ofrece otros elementos que -modelados (perfilados) de una manera adecuadapueden facilitar su función focalizadora con el valor de unicidad: la negación y la discontinuidad (esta última común de ambos vocablos). Para explicar la nueva situación será necesario remitirse a la lingüística cognitiva (tercer nivel del análisis) y, más precisamente, al Principio de Iconicidad (Givón, 1990). De acuerdo con este principio, tal situación es posible si ubicamos el adverbio analizado en la posición antepuesta al elemento focalizado (Givón, 1990: 65-66).

Visto que la lengua es una herramienta lineal que debe servirnos para expresar el universo multidimensional en el cual vivimos, la regla de iconicidad nos permite expresar las secuencias de eventos, así como los vemos y motiva una extensión de estas relaciones a las dimensiones más abstractas, como por ejemplo las cualidades y los sentimientos. En la visión del mundo europea, lo primero se asocia siempre con lo mejor, lo más fuerte y lo más importante. Además, en este universo la dirección de los cambios va siempre de izquierda a derecha. Así las cosas, de acuerdo con el Principio de Iconicidad, el hecho de anteponer nomás no tiene solo consecuencias sintácticas, sino que lleva consigo unas consecuencias semántico-pragmáticas. En primer lugar, habida cuenta de la estructura nocional del adverbio (negación + discontinuidad), la posición principal -la más fuerte en el enunciado- determina el estatus semántico-comunicativo de nomás y todos los elementos que entren en su alcance, ubicándolos automáticamente en la parte remática del enunciado: nomás, al igual que sólo, no puede constituir el tema del enunciado y permite desprenderlo del valor de adición. Sin embargo, de todo ello puede surgir la pregunta por el estatus de nomás como marcador del contraste. Observemos, que el fenómeno de contrastividad enunciativa opera en el área de la estructura temático-remática. Como es bien sabido, un enunciado contrastivo tiene cualidades diferentes 
respecto a aquel que simplemente trae una información nueva. El enunciado que aporta una información nueva se compone de una parte temática (explícita o no) y de otra remática, que comprende esta información. La tarea básica del enunciador al formular un enunciado contrastivo es, sin embargo, escoger al candidato para el foco de contraste entre los elementos que constituyen este enunciado y asignarle un marcador que lo resalte, porque ambos elementos contrastados pertenecen a la parte temática. No obstante, en el caso de nomás en la posición antepuesta no estamos ante la elección entre dos posibilidades presentadas, sino ante "una lectura de unicidad, [que] niega todas las alternativas posibles en el universo del discurso» (Gutiérrez-González, 2011: 626). Pero ¿es seguro que sea así?, o bien ¿el adverbio nomás antepuesto desempeña en los enunciados españoles la misma función que /-lla/ cuando focaliza el elemento en su alcance «como si el resto de las cosas que puedan afectar a [lo denotado] en concreto no importaran»? (Calvo Pérez, 2000). Ya Kany (1976) demostró que los valores de nomás varían según su posición en el enunciado. Además, se observa una tendencia de posponer este adverbio a la parte constituyente del enunciado. En los corpus estudiados es mucho más difícil encontrar los ejemplos con nomás antepuesto. El cambio de posición conlleva una modificación del significado.

Ahora bien, la Academia describe todos los demás valores de nomás como "énfasis». Sin embargo, como también observa con razón Calvo, comentando la misma etiqueta otorgada a los valores de las partículas /-lla/ y /-k(y)/, la denominación de énfasis es «una solución cómoda para un conjunto de posibilidades» (Calvo Pérez, 2000: 101). Entre estas posibilidades interpretativas de los morfemas indígenas, Calvo enumera:

el afecto: warmicha-illa-yña-kuni = quiero a mi mujercita, la cortesía: wathiya ruwasqata, p'aruranpu-lla-yña = para hacer guatía, rompe ya los terrones [por favor] y su par expresivo, la descortesía: imatan muyupayamuwashanki? Rimari-lla-yña = ¿qué haces con tantos preámbulos?, habla de una vez» (Calvo Pérez, 2000: 101).

Recordemos ahora que Cusihuamán (1976) además de ya analizado significado de limitación de /-lla/ destaca otro que «denomina valor de intensificación con la traducción en no más, siempre, usualmente, por lo general» (Cusihuamán, 1976: 255, citado por Calvo Pérez, 2000: 101). 
Ambos valores presentados pueden ser traducidos con nomás, aunque sólo el primero, el más restrictivo, exige la posición antepuesta del adverbio analizado.

Pasemos, pues, a los valores de nomás en posposición. No obstante, a nuestro parecer, es muy importante añadir aquí la observación de Gutiérrez-González según la cual el valor de intensificación de nomás está vinculado con el uso de nomás como modificador de construcciones adverbiales (adverbios de lugar o de manera). La autora ofrece la siguiente comparación del valor de nomás pospuesto a estos adverbios con el estrictamente acotador que este vocablo adquiere siempre al anteponerse a ellos: el valor enfático (Gutiérrez-González, 2011: 42):

(3) El supermercado está acá nomás. > aquí mismo

(4) ¿Así nomás? ¡Qué fácil! > simplemente / meramente

(5) Siempre compro la fruta nomás acá.

(6) Me dijo que podría nomás ahora

(7) Ahoralya nomás me peino y salgo > ahora mismo

Este valor enfático es también, como tantos otros, motivado cognitivamente. En el caso de los adverbios de lugar, nomás antepuesto expone la acotación del lugar denotado; en el caso de los adverbios de tiempo, estamos ante una extensión metafórica del significado típica, que ahora resalta la «acotación» de un lapso de tiempo, etc.

No obstante, al desplazar nomás a la posición icónicamente menos privilegiada, suavizamos su valor restrictivo, que evoluciona de lo esencial/lo preciso a lo generalizado. No en vano, en las explicaciones del valor enfático de nomás este vocablo suele reemplazarse con simplemente, cuyo valor aquí es una extensión del significado de otro exponente natural (aunque ya no prototípico, como lo es el caso de solo) de la unicidad: simple. En su acepción primaria, simple significa formado por un solo elemento o por pocos; y en la secundaria, sencillo, no compuesto > no complicado. Sin embargo, el primer paso en la evolución del sentido de nomás es el cambio de las funciones discursivas del vocablo (Gutiérrez-González, 2011: 42):

(8) Llovió nomás > Creía que (no) iba a llover y llovió nomás 
En este uso la función de nomás es inferencial puesto que, mediante el modelado (la perfilación) ${ }^{11}$ de una combinación de los conceptos de negación+discontinuidad (ver supra), el adverbio indica que lo denotado en el enunciado ha ocurrido en contra de algunas expectativas.

El paso siguiente es el modelado (la perfilación) del concepto de discontinuidad en función aspectual limitativa que conlleva la función discursiva de incisión / estimulación. Esta función se parece a la que en los enunciados similares desempeñan las construcciones y punto/y ya está. Son valores que podríamos denominar estimuladores, de los cuales se desprenden los concesivos y luego el valor de cortesía:

Los valores estimuladores > concesivos (Gutiérrez-González, 2011: 42):

(9) ¡Dale nomás, sin miedo!

(10) Pregunta nomás > pregunta todo lo que quieras

En ambos ejemplos alegados el vocablo nomás refuerza el valor concesivo del predicado principal y puede ser sustituido por simplemente

Los valores de cortesía (Gutiérrez-González, 2011: 42):

(11) ¿Qué no más has traído?

(12) Pasen nomás, pasen, entren, no se queden ahí parados [...]

Los valores de cortesía parecen los más diferentes al español estándar. En el ya mencionado "foro» (en línea) leemos una discusión muy animada sobre la interpretación de nomás que resumimos aquí sirviéndonos de las palabras de uno de los foreros: «[...] lo corriente es que un español al oír ese nomás se quede extrañado. [...] La frase, "pasen nomás", a mí me la dicen al abrir la puerta y ya dudo si en realidad me deja o no entrar [...]》.

${ }^{11}$ De acuerdo con la idea de Langacker (1987: §5.1), la perfilación o el modelado semántico-discursivo permite destacar uno de los elementos de una estructura conceptual dada: «El perfil, por su lado, es la subestructura destacada sobre la base que la expresión en cuestión designa conceptualmente». 
La mayoría de los estudiosos están de acuerdo en que el uso de nomás con el valor de cortesía está relacionado con el valor analógico de /-lla/ quechua. No obstante, teniendo en cuenta todos los factores culturales, sociológicos y pragmalingüísticos, el análisis de la evolución de nomás hacia la función del marcador de cortesía requiere un estudio separado. Aquí sólo nos limitaremos a decir que uno de los factores que pueden servir de andamiaje para tal estudio lo constituye la presencia del concepto de discontinuidad y el valor inferencial del vocablo cuyo ámbito y dirección se forman según el amplio contexto discursivo, cultural, etc. Esta es una explicación posible del uso del vocablo como tal. El valor pragmático particularizado, sin embargo, puede modificarse según el tipo de construcción lingüística a la cual el adverbio modifica, pero, ante todo, según las condiciones del discurso. Lo mismo ocurre en el caso de los usos con el significado de descortesía. Es el universo discursivo que condiciona tanto el significado irónico como el de amenaza. Veamos los ejemplos de los valores que se desplazan al polo de la descortesía:

- el irónico:

(13) Sigue así nomás sin arreglar el problema, total para eso estamos el resto

- el de amenaza:

(14) ¡Sigue molestando nomás!, ya te daré tu merecido

\section{CONCLUSIONES}

Como ya hemos mencionado, compartimos la hipótesis de una fuerte influencia del uso de /-lla/ en el desarrollo de los valores de nomás. No podemos olvidar, sin embargo, que existe un número impresionante de valores del morfema quechua que en gran parte no se expresan mediante nomás (Calvo Pérez, 2000: 101 n. 32). El empleo del aparato de la lingüística cultural nos ha permitido analizar la estructura conceptual representada por el adverbio nomás y las motivaciones cognoscitivas de su adaptación, así como proponer una vía de su evolución semántico-discursiva. En primer lugar, la descomposición semántica nos ha permitido descubrir los conceptos simples, universales (de negación 
y de discontinuidad) los cuales fueron el elemento común más importante entre la estructura conceptual de construcción lingüística castellana de no más y la del morfema quechua /-lla/, factor que predestinó a la construcción castellana a su futura evolución. El paso siguiente, el análisis pragmático (presuposición-inferencia) ha explicado el origen del valor inferencial del nomás americano (ejemplo 8). El tercer paso, el análisis cognitivo, ha completado la imagen de la evolución de nomás con informaciones inestimables para explicar un importante cambio del valor de nomás debido al desplazamiento de su posición en la oración, contribuyendo de este modo a obtener una imagen más completa de dicha evolución.

En definitiva, gracias a la aplicación de una metodología multidimensional, hemos demostrado que todos los valores de nomás andino están fuertemente relacionados con la estructura nocional básica del vocablo y especialmente con distintos modelados del concepto de discontinuidad. Además, nuestro análisis ha permitido advertir que los valores del adverbio en cuestión "compartidos» con el morfema quechua /-lla/ no podrían haberse desarrollado si la estructura interna de nomás no lo hubiera facilitado.

\section{BIBLIOGRAFÍA CITADA}

AdELAAR, W.F. (1977): Tarma Quechua, grammar, texts, dictionary. Lisse, The Peter de Ridder Press.

ANUSIEWICZ, J. (1994): Lingwistyka kulturowa. Zarys problematyki. Wrocław, Uniwersytet

Wrocławski.

BOGACKI, K. y KAROLAK, S. (1991): «Fondements d'une grammaire à base sémantique». Lingua e Stile, XXVI.3, págs. 309-345.

Calvo Pérez, J. (2000): «Partículas en castellano andino». En: Calvo Pérez, J. (ed.): Teoría y práctica del contacto: el español de América en el candelero. Madrid, Iberoamericana, págs.73-112.

Cerrón Palomino, R. (2004): Castellano andino: aspectos sociolingüísticos, pedagógicos y gramaticales. Lima, PUCP.

CUENCA, M.J. y HiLfERTY, J. (1999): Introducción a la lingüística cognitiva. Barcelona, Ariel. 
Cusihuamán, A. (1976): Gramática Quechua Cuzco-Collao. Cuzco, Centro de Estudios Regionales Andinos «Bartolomé de las Casas».

DE GRANDA, G. (1999): Español y lenguas indoamericanas en Hispanoamérica. Estructuras, situaciones y transferencias. Valladolid, Universidad de Valladolid.

(2001): Estudios de lingüística andina. Lima, PUCP.

GIVón, T. (1984): Syntax: A Functional-Typological Introduction, vol. I. Amsterdam-Philadelphia, John Benjamins.

GRZEGORCZYKOWA, R. (1999): «Profilowanie a inne pojęcia opisujące hierarchiczną strukturę znaczenia». En Bartmiński, J. y Tokarski, R. (eds.): Profilowanie $w$ języku $i$ w tekście. Lublin, UMCS, págs. 2-11.

Guillaume, G. (1970): Temps et verbe. Théorie des aspects, des modes et des temps. Paris, Champions.

GuTIÉRREZ GONZÁLEZ, Y.M. (2011): “¿Operadores focales exhaustivos nomás?». Cuadernos de la $A L F A L, 3$, págs. $42-59$ (en línea: <http://www.linguistical fal.org/03_cuaderno_006.pdf> [consulta: 12 de febrero de 2019]).

KANY, C.E. (1976): Sintaxis hispanoamericana. Madrid, Gredos.

KAROLAK, S. (1994): "Le concept d’aspect et la structure notionnelle du verbe».

En: Studia Kognitywne, vol. I. Varsovia, Slawistyczny Ośrodek Wydawniczy, págs. 21-41.

LANGACKER, R.W. (1987): Foundations of Cognitive Grammar, vol. I: Theoretical Prerequisites. Stanford, Stanford University Press.

LiUzzI, S. y KIRTCHUK, P. (1989): «Tiempo y aspecto en guaraní». Amerindia, 14 , págs. 9-42.

Palmer, G.B. (2000): Lingüística cultural. E. Bernárdez (trad.). Madrid, Alianza Editorial.

RAE = REAL ACADEMIA EsPAÑOLA (2019): Diccionario de la Lengua Española (en línea: <https://dle.rae.es> [consulta: 07 de mayo de 2020]).

Tovar, A. (1964). «Español y lenguas indígenas. Algunos ejemplos». En: Presente y Futuro de la Lengua Española. Madrid. Ediciones Cultura Hispánica, vol. II, págs. 245-257.

WIERzBICKA, A. (1988): The semantics of grammar. Amsterdam, John Benjamins.

(1991): Cross-Cultural Pragmatics. Berlin, Mouton De Gruyter.

WILK-RACIĘSKA, J. (2007): "Nuestro mundo, nuestras visiones del mundo y las lenguas que lo describen todo...». Anuario de Estudios Filológicos, XXX, págs. 439-453. 
(2009): Od wizji świata do opisu językoznawczego w kategoriach lingwistyki kulturowej. Uwagi na temat opisu języka hiszpańskiego. Katowice, Wydawnictwo Uniwersytetu Śląskiego.

(2012): Entre la visión del mundo temporal y la aspectual. Casos del español sudamericano. Katowice, Wydawnictwo Uniwersytetu Śląskiego.

_ (2016): «La Lingüística cultural: una aportación a la metodología». En Wilk-Racięska, J. et al. (eds.): Relecturas y nuevos horizontes en los estudios hispánicos. Katowice, Wydawnictwo Uniwersytetu Śląskiego, págs. 205-217.

(2018): «Análisis conceptual de los valores del gerundio en el español peruano: una propuesta metodológica». En Risco, R. (coord.): Estudios de variación y contacto lingüístico en el español peruano. La Plata, UNLP, págs. 81-110.

(2020a): «La investigación científica del español sudamericano: consideraciones sobre la selección de criterios». Lublin Studies in Modern Languages and Literatur, 44.3, págs. 80-91.

(2020b): «Sobre los pares aspectuales en polaco: un acercamiento a la aspectualidad eslava». Revista Española de Lingüística Aplicada (en prensa).

Joanna WILK-RACIĘSKA Universidad de Silesia joanna.wilk-racieska@us.edu.pl 0000-0002-9183-3766 
